\title{
Evaluación de Trichoderma sobre hongos contaminantes de semillas de palma híbrida interespecífica OxG (Elaeis oleifera $\mathbf{x}$ Elaeis guineensis)
}

\section{Evaluation of Trichoderma on contaminant fungi of interspecific hybrid seed palm OxG} (Elaeis oleifera $\mathrm{x}$ Elaeis guineensis)

\section{Avaliação de Trichoderma sobre fungos contaminantes de sementes de palma híbrida interespecífica OxG (Elaeis oleifera x Elaeis guineensis)}

\author{
Oscar Eduardo Ladino-Rey ${ }^{1}$, Gabriel Arturo Chaves-Betancourt² ${ }^{2}$ José David Rubio-Gómez ${ }^{3}$, \\ Christian Andrei Chacin-Zambrano ${ }^{4}$
}

Forma de citar: O.E. Ladino-Rey, G.A. Chaves-Betancourt, J.D. Rubio-Gómez y C.A. Chacin-Zambrano, "Evaluación de Trichoderma sobre hongos contaminantes de semillas de palma híbrida interespecífica OxG (Elaeis oleifera x Elaeis guineensis)", Respuestas, vol. 22, no. 1, pp. 96-102, 2017.

Recibido:

Junio 30 de 2016

Aceptado: octubre 14 de 2016

\section{Resumen}

El proceso de germinación de semillas de palma hibrida interespecífica OxG (E. oleifera $\mathrm{x}$ E. guineensis) de la empresa Indupalma ltda ha presentado una disminución en la producción, lo que se ve reflejado en pérdidas económicas, debido a la existencia de hongos filamentosos que afectan las semillas. Estudios previos evidenciaron el potencial controlador de Trichoderma sp. sobre estos hongos. En esta investigación se evaluaron cepas nativas y comerciales de T. harzianum y $T$. viride frente a hongos contaminantes prevalentes de semillas de palma hibrida interespecífica. Se aislaron e identificaron once hongos patógenos prevalentes a partir de semillas de palma hibrida interespecífica que fueron eliminadas de las diferentes etapas del proceso de germinación. Se llevaron a cabo pruebas de plato dual a los hongos contaminantes seleccionados y se determinó el porcentaje de inhibición de crecimiento radial (PICR) y micoparasitismo. Estos hongos fueron inhibidos en su crecimiento por el efecto antagonista de T. harzianum y T. viride. De igual manera T. viride comercial evidenció gran potencial biocontrolador al inhibir por encima del $60 \%$ a siete de las once cepas de hongos contaminantes.

Palabras clave: Antagonismo, germinación de semillas, PICR.

\section{Abstract}

The germination process of hybrid palm interspecific OxG (E. oleifera $x$ E guineensis.) seeds in the company Indupalma ltda has filed a decrease in production, which is reflected in economic losses due to the existence of filamentous fungi that affect seeds. Previous studies have shown the potential controller Trichoderma sp. on these fungi. Commercial and native strains of $T$. harzianum and T. viride were evaluated against prevalent fungal contaminants of interspecific hybrid palm seeds. They were isolated and identified eleven strains of pathogenic fungi from interspecific hybrid palm seeds that were removed from the different stages of germination. Dual plate tests were conducted at prevalent fungi and the percent inhibition of radial growth (PICO) and mycoparasitism were determined. These fungi were inhibited in growth by the antagonistic effect of $T$. harzianum and $T$. 
viride. Similarly, T.viride showed a great potential as a biocontrol agent to inhibit over $60 \%$ seven out of eleven strains of contaminating fungi.

Keyword: Antagonism, seed germination, PICR.
Enero - Junio 2017

ISSN 0122-820X

E-ISSN 2422-5053

PP: 96-102

\section{Resumo}

O processo de germinação de sementes de palma hibrida interespecífica OxG (E. oleifera $\mathrm{x}$ E. guineensis) da empresa Indupalma Ltda., tem apresentado uma diminuição na produção, que se reflete em perdas económicas, devido à existência de fungos filamentosos que afetam as sementes. Estudos prévios evidenciaram o potencial controlador de Trichoderma sp. sobre estes fungos. Nesta pesquisa cepas nativas e comerciais de $T$. harzianum e T. viride foram avaliadas frente a fungos contaminantes prevalentes de sementes de palma hibrida interespecífica. Foram isolados e identificados onze fungos patógenos prevalentes a partir de sementes de palma hibrida interespecífica que foram eliminadas das diferentes etapas do processo de germinação. Foram realizados tests de placa dupla aos fungos contaminantes selecionados e se determinou a porcentagem de inibição de crescimento radial (PICR) e micoparasitismo. Estes fungos foram inibidos em seu crescimento pelo efeito antagonista de T. harzianum e T. viride. Da mesma forma $T$. viride comercial evidenciou grande potencial biocontrolador, inhibindo a mais do $60 \%$ das onze cepas fùngicas contaminantes.

Palavras-chave: Antagonismo, germinação de sementes, PICR.

\section{Introducción}

Indupalma LTDA es una empresa dedicada al cultivo, extracción de aceite y producción de semillas de palma híbrida interespecífica OxG (E. oleifera x E.guineensis) [1]. Uno de los productos de mayor auge y demanda son los embriones de semilla híbrida, el cual pasa por un proceso desde fruto carnoso hasta obtener semillas germinadas.

Actualmente, este proceso ha sido afectado por la presencia de hongos, la cual se presenta en las etapas de calefacción, germinación y conservación de embriones, repercutiendo en pérdidas económicas, al disminuir el porcentaje de germinación, destruiry consumir el embrión de la semilla germinada y no germinada, o estar en el embrión y consumir el endospermo [2]. Como contaminantes del proceso de germinación de semillas, se aislaron hongos de los géneros: Aspergillus sp., Rhizopus stolonifer, Fusarium sp., Mucor sp. y Penicillium sp., y donde la aplicación de fungicidas no causaron control efectivo sobre ellos [3],[4]. Sin embargo, la aplicación de Trichoderma sp., un hongo conocido por su capacidad antagónica y micoparasítica [5], logró inhibir los hongos presentes.

Por lo anterior, este estudio evaluó el efecto antagónico de diferentes cepas de Trichoderma sp. contra hongos contaminantes en el proceso de germinación de semillas de palma híbrida interespecífica $\mathrm{OxG}$ (E. oleifera x E.guineensis).

\section{Materiales y métodos}

El estudio se realizó en el laboratorio agronómico $\mathrm{y}$ en el germinador del departamento de investigación y desarrollo de la empresa indupalma ltda, ubicada en el km 10 via al mar, San Alberto, Cesar, Colombia $07^{\circ} 47^{\prime} 42^{\prime \prime}$ y $07^{\circ} 36^{\prime} 45^{\prime \prime}$ de $1 \mathrm{n}, 73^{\circ}$ $23^{\prime} 33^{\prime \prime}$ y $73^{\circ} 34^{\prime} 48^{\prime \prime}$ de 1 o de Greenwich. La plantación se encuentra ubicada a $120 \mathrm{msnm}$, con humedad relativa del $75 \%$ y temperatura media ambiental de $27 \pm 5^{\circ} \mathrm{C}$. 
Las muestras fueron las semillas de palma híbrida interespecífica $\mathrm{OxG}$ eliminadas del proceso de germinación de semillas por tener contaminación fúngica superficial. El muestreo se realizó al azar, tomando semillas de cada etapa del proceso en un intervalo de 20 a 30 días entre cada toma de muestra y de diferentes lotes. En cada toma de muestra, se seleccionaron 50 semillas en la etapa de Calefacción, 200 de germinación y 100 de conservación de embriones.

\subsection{Aislamiento e identificación de hongos contaminantes}

Las semillas fueron raspadas con un asa bacteriológica en la zona afectada, principalmente en el opérculo. Se sembró en cajas de Petri con medio de cultivo papa-dextrosa- agar (PDA) por triplicado; se incubaron a $25^{\circ} \mathrm{C}$ durante siete días o el tiempo que requirió cada cepa. Se repicaron de nuevo en cajas de Petri con medio de cultivo PDA para obtener cepas puras.

Se observaron los hongos en microscópio de luz a 40 y $100 \mathrm{X}$ con azul de lactofenol o hidroxido de Potasio. Además, los morfotipos fueron identificados mediante claves taxonómicas de [6].

Para la evaluación del efecto antagónico se seleccionaron aquellos hongos contaminantes que presentaron prevalencia mayor a $2 \%$ en alguna de las etapas del proceso de germinación de semillas o aquellos reportados en estudios anteriores realizados [4].

\subsection{Evaluación del efecto antagónico de las cuatro cepas de Trichoderma.}

La evaluación del efecto antagónico se realizó con dos cepas comerciales T. harzianum (TharC) y T. viride (TvirC) y dos nativas, la primera T. harzianum (TharN) obtenida a partir de suelo de E. oleifera y la segunda T. viride (TvirN) fue aislada a partir de espigas masculinas de E. guineensis. Se usó la metodología de plato dual [7]. Las cepa del hongo antagonista se enfrento contra los hongos prevalentes aislados y fueron seleccionados aquellos hongos contaminantes que presentaran una prevalentia superior al $2 \%$ en alguna de las etapas del proceso. Se sembró a $60 \mathrm{~mm}$ de distancia en la caja Petri con medio de cultivo PDA. Se tomó medida del radio diariamente durante ocho días. Se incubó a $30^{\circ} \mathrm{C}$. Cada ensayo se realizó cinco veces.

En la ecuación (1) Se estudiaron los siguientes parámetros: Radio de Crecimiento Antagonista (RCA), Radio de crecimiento hongo aislado (RCP), Micoparasitismo (MICMO) y Porcentaje de Inhibición de Crecimiento Radial (PICR). La competencia por nutrientes y espacio se valoró comparando la velocidad de crecimiento del aislado fungico (RCP) y de los antagonistas (RCA) [7]. Para la evaluación del porcentaje de inhibición de crecimiento radial (PICR) (Ecuacion 1) se empleó la fórmula donde $\mathrm{R} 1$ es el radio del patógeno testigo y $\mathrm{R} 2$ es el radio del patógeno en enfrentamiento [8].

$$
P I C R=\frac{(R 1-R 2)}{R 1} \times 100
$$

Para evaluar el micoparasitismo se usó la escala creada por [8] para la evaluación de la capacidad micoparasítica, de acuerdo a la medida de la invasión de la superficie, colonización y esporulación de Trichoderma sp. sobre los hongos donde se presente crecimiento sobre el micelio del patógeno.

Los datos se analizaron mediante un análisis de varianza. En los casos donde se observaron diferencias significativas se realizó la prueba de Tukey $(\mathrm{P} \leq 0,05)$ como comparador de medias entre tratamientos. El análisis se realizó mediante el software estadístico Minitab ${ }^{\circledR} 16$, hecho por Minitab Inc. 


\section{Resultados y análisis}

Los hongos encontrados en cada una de las fases no difieren a los aislados en otros reportes [4]. Sin embargo, presentando las mismas condiciones, no se aislaron hongos del género como Sclerotinia sp., o especies de Aspergillus como A. clavatus o A. ochraceus que anteriormente fueron reportados por dichos autores. Cabe mencionar que se encontraron hongos como A. flavus, o Penicillium, que no entran dentro de los hongos reportados en este estudio, debido a que su presencia en las muestras fue esporádica $(<2 \%)$. Schizophyllum sp. y Syncephalastrum sp., son los mayores contaminantes en las muestras obtenidas (Tabla I), a pesar de ello, no se presentaron en ensayos anteriores [4]. Esto puede mostrar que existe posiblemente alguna rotación o disposición de los hongos, con respecto al tiempo o inclusión de los mismos desde campo.

Tabla I. Los hongos y otros microorganismos aislados de las diferentes etapas del proceso

\begin{tabular}{|c|c|c|c|}
\hline Microorganismo & $\mathrm{C}(\%)$ & G (\%) & E (\%) \\
\hline A. flavus & 0,00 & 1,35 & 0,00 \\
\hline A. fumigatus & 0,00 & 2,20 & 3,03 \\
\hline A. niger & 10,17 & 3,75 & 0,00 \\
\hline A. terreus & 8,33 & 10,00 & 2,82 \\
\hline Basipetospora sp. & 0,00 & 0,00 & 0,63 \\
\hline Fusarium sp. & 0,00 & 1,90 & 10,29 \\
\hline Fusarium sp. & 0,00 & 0,99 & 0,00 \\
\hline Fusarium sp. & 0,00 & 0,17 & 1,90 \\
\hline Mucor sp. & 54,02 & 16,66 & 10,35 \\
\hline Penicillium sp. & 0,00 & 2,07 & 0,63 \\
\hline Penicillium sp. & 5,70 & 1,16 & 0,63 \\
\hline Penicillium sp. & 0,00 & 0,79 & 0,32 \\
\hline Penicillium sp. & 0,6 & 0,70 & 0,00 \\
\hline Rhizopus stolonifer. & 4,40 & 4,95 & 0,00 \\
\hline Schizophyllum sp. & 11,62 & 26,03 & 52,29 \\
\hline Syncephalastrum sp. & 0,00 & 18,41 & 4,22 \\
\hline T. harzianum & 0,00 & 0,19 & 0,00 \\
\hline T. viride & 0,00 & 4,83 & 0,73 \\
\hline Bacterias & 2,52 & 0,00 & 0,41 \\
\hline Levaduras & 2,63 & 3,86 & 11,74 \\
\hline
\end{tabular}

Los hongos Fusarium sp., Aspergillus sp., Penicillium sp. y Schizophyllum sp. se presentan como contaminantes de semillas de palma de aceite. Schizophyllum sp. es conocido como contaminante de semillas de palma de aceite y logra disminuir la tasa de germinación por debajo del $65 \%$ y es prevalente en todo el proceso del 11-52\% [9],[10].

El efecto antagónico de T. harzianum y T. viride sobre los hongos contaminantes aislados seleccionados (Tabla II). 
Evaluación de Trichoderma sobre hongos contaminantes de semillas de palma híbrida interespecífica OxG (Elaeis oleifera $x$ Elaeis guineensis)

Tabla II. Valores de PICR obtenido de las cuatro cepas de Trichoderma sp. sobre hongos contaminantes prevalentes.

\begin{tabular}{|c|c|c|c|c|c|c|c|c|c|c|c|c|c|}
\hline \multirow[b]{2}{*}{ Aspergillus fumigatus } & \multirow[b]{2}{*}{ IPS01 } & \multicolumn{3}{|c|}{ TharC } & \multicolumn{3}{|c|}{ Thar $\mathrm{N}$} & \multicolumn{3}{|c|}{ TvirC } & \multicolumn{3}{|c|}{ Tvir $\mathrm{N}$} \\
\hline & & 55 & b & III & 50 & $\mathrm{~b}$ & I & 62 & a & IV & 59 & a & IV \\
\hline Aspergillus niger & IPS02 & 47 & a & 0 & 18 & $\mathrm{c}$ & 0 & 43 & $\mathrm{a}$ & I & 35 & b & IV \\
\hline Aspergillus terreus & IPS03 & 57 & a & 0 & 44 & $\mathrm{c}$ & 0 & 61 & a & 0 & 61 & b & II \\
\hline Fusarium sp. & IPS04 & 69 & $\mathrm{c}$ & 0 & 68 & $\mathrm{c}$ & 0 & 85 & a & IV & 76 & b & IV \\
\hline Fusarium sp. & IPS05 & 30 & b & 0 & 24 & b & 0 & 59 & a & IV & 52 & $\mathrm{a}$ & II \\
\hline Mucor sp. & IPS06 & 66 & $\mathrm{c}$ & I & 60 & $b$ & 0 & 70 & $a b$ & IV & 71 & a & II \\
\hline Penicillium sp. & IPS07 & 25 & b & 0 & 20 & $b$ & 0 & 43 & a & III & 47 & $\mathrm{a}$ & IV \\
\hline Penicillium sp. & IPS08 & 23 & $\mathrm{~b}$ & 0 & 16 & $\mathrm{~b}$ & 0 & 52 & a & IV & 43 & a & 0 \\
\hline Rhizopus stolonifer & IPS09 & 37 & $\mathrm{a}$ & IV & 32 & $b$ & III & 39 & $\mathrm{a}$ & IV & 31 & b & IV \\
\hline Schizophyllum sp. & IPS10 & 43 & $\mathrm{c}$ & 0 & 34 & d & 0 & 65 & a & I & 53 & b & 0 \\
\hline Syncephalastrum sp. & IPS11 & 66 & $\mathrm{~b}$ & 0 & 60 & $\mathrm{c}$ & 0 & 80 & $\mathrm{a}$ & IV & 73 & $\mathrm{a}$ & IV \\
\hline
\end{tabular}

Las medias seguidas por la misma letra en la fila no difieren entre si por la prueba de Tukey $(\mathrm{P}<0,05)$

Grados de escala MICMO: 0: No invasión de superficie de patógeno; I: Invasión del 25\% de la superficie del patógeno; II: Invasión del 50\% de la superficie del patógeno; III: Invasión total de la superficie del patógeno; IV: Invasión total y esporulación sobre la superficie del patógeno.

Al comparar el efecto antagónico de las cuatro cepas de Trichoderma frente a los hongos contaminantes seleccionados se observan diferencias significativas en todos los tratamientos $(\mathrm{P}=0.0)$. El tratamiento TvirC fue el mayor controlador de hongos, inhibiendo el crecimiento de seis de los once hongos estudiados en por lo menos el $60 \%$ de PICR, el cual es el valor determinado para una cepa promisoria de uso como antagonista [11], además micoparasitó y esporuló sobre la superficie de ocho cepas de hongos contaminantes estudiados.

Se observa grandes diferencias entre los valores de PICR para los enfrentamientos entre las cuatro cepas de Trichoderma y Fusarium sp., demostrando la necesidad de especificidad del hongo antagonista sobre el hongo fitopatógeno. Este enfrentamiento ha evidenciado valores de PICR promisorios en aislamientos realizados en maíz (73\%) [12] y Maracuya (65\%) [7].

Ensayos in vitro de Trichoderma pueden presentar inhibición y micoparasitismo sobre Schizophyllum sp. el cual hace parte de los hongos alimentados de madera en descomposición, sin embargo, estudios han demostrado que esta actividad disminuye al realizar ensayos in vivo sobre bloques de madera [13],[9].

Algunos géneros como Mucor sp., Syncephalastrum sp. y Rhizopus sp. no están documentados como patógenos de semillas de palma; sin embargo, estos se aislaron con frecuencia en las muestras tomadas, siendo Syncephalastrum sp. uno de los hongos que se presentó en las tres fases estudiadas.

\section{Conclusiones}

Las cuatro cepas de Trichoderma sp. inhibieron el crecimiento de los hongos contaminantes de semillas de palma híbrida interespecífica.

La cepa de $T$. viride comercial es la más promisoria para el uso como antagonista, debido a que inhibe en gran medida al menos $60 \%$ de PICR en seis de los once hongos estudiados. Además, esta cepa presentó micoparasitismo en diez de los once hongos evaluados y en ocho de ellos colonizó y esporuló la mitad o más del micelio del patógeno. 


\section{Referencias}

[1] Indupalma, "Informe de sostenibilidad 2011”, 2001. [En línea]. Disponible en: http:/www.indupalma.com/sites/ default/files/file_attach/Informe $\% 20$ Sostenibilidad $\% 20$ INDUPALMA $\% 20$ 2010-2011.pdf.

[2] S. Mora, C. Chinchilla, A. Sanchez y R. Escobar, "Innovación en los procesos para mejorar la calidad de las semillas germinadas y de las planturas de palma de aceite". Palmas, vol. 28. no. especial, tomo 1, pp. 265-272, 2007.

[3] A. Sanchez Potes, "Enfermedades de la palma de aceite en América Latina". Palmas, vol 1, no. 4, pp. 5-38, 1990.

[4] A. Corena, C. Zambrano y A. Gualdron, "Evaluación in vitro de fungicidas para el control de hongos patógenos en semillas hibridas Elaeis guineensis jacq x Elaeis oleifera Bailey, en el germinador de Indupalma ltda. San Alberto, Cesar". Tesis de Grado Profesional Microbiologia Industrial, Facultad de Ciencias Exactas Físicas y Naturales, Universidad de Santander, Bucaramanga, 2011.

[5] M. Chavez, "Produccion de Trichoderma sp y evaluacion de su efecto en cultivo de crisantemo (Dendranthema grandiflora)", Tesis de Grado Profesional Microbiologia Indutrial, Pontificia Universidad Javeriana, Bogota DC, 2006.

[6] H.L. Barnett and B.B. Hunter, Illustrated Genera of Imperfect Fungi, Fourth Edition, St. Paul, Minnesota, USA: The American Phytopathological Society, 1998.

[7] R.J Fernandez Barbosa y C.L Suarez Meza, "Antagonismo in vitro de Trichoderma harzianum Rifai sobre
Fusarium oxysporum Schlechr F. sp passiflorae en Maracuya (Passiflora edulis Sims var. Flavicarpa) del municipio zona bananera colombiana". Revista Facultad Nacional Agronómica de Medellin, vol 62, no 1, pp. 47434748, 2009.

[8] M. Ezziyani, S. Perez, M. Requena, L. Rubio y M. Candela, "Biocontrol por Streptomyces rochei de la podredumbre del pimiento (Capsicum annuum L.) causada por Phytophora capsici", Anales de Biologia, vol 26, pp. 69-78, 2004.

[9] A. Dikin, K. Sijam, Z.A Mior Ahmad, and I. Abu Sean, "Biological Control of Seedborne pathogen of oil palm, Schizophyllum commume Fr. With antagonistic Bacteria”, International Journal of Agriculture \& Biology, vol. XX, pp. 507-512, 2003.

[10] T. Agarwal, A. Malhotra, M. Biyani, and P. Trivedi, "In vitro interaction of Trichoderma isolates against Aspergillus niger, Caetomium sp. and Penicillium sp", Indian Journal of Fundamental and Applied Life Sciences, vol. 1, no 3, pp. 125-128, 2011.

[11] R. Noveriza and T.H Quimio, "Soil Mycoflora of black pepper rhizosphere in the philippines and their in vitro antagonism against Phytophthora capsici L", Indonesian Journal of Agricultural Sciences, vol. 5, no. 1, pp. 1-10, 2004.

[12] S. Suhaida, and M.Z Nur AinIzzati, "The efficacy of Trichoderma harzianum T72s as a biocontrol agent of Fusarium ear rot desease of maize". International Journal of Agriculture and Biology. vol. 15, pp. 1175-1180, 2013.
Enero - Junio 2017 ISSN 0122-820X

E-ISSN 2422-5053 PP: 96-102 
[13] T. Highley and J. Ricard, "Antagonism of Trichoderma spp. and Gliocladium virensagainstwood decayfungi", Material und Organismen, vol. 23, pp. 157- 169, 1988. 